\title{
Interoperabilidade entre dispositivos como serviços: uma abordagem interdisciplinar
}

\author{
Daniela Barreiro Claro ${ }^{12}$, Luis Emanuel Neves de Jesus ${ }^{1}$, Alexandre Silva Gomes ${ }^{2}$, \\ Roberto de Cerqueira Figueiredo ${ }^{1}$, Mariane Dourado Correia ${ }^{2}$, Cleiton Fernando \\ Lima Sena ${ }^{1}$, Rafael Glauber ${ }^{1}$, Wellington Assunção Azevedo ${ }^{2}$, José Alejandro \\ Moreno Alfonzo ${ }^{2}$, Luan Silva Santana ${ }^{2}$, Aydin Jadidi \\ ${ }^{1}$ FORMAS Research Group - LaSiD - PGComp \\ Departamento de Ciência da Computação, Universidade Federal da Bahia \\ Av. Adhemar de Barros, s/n, Ondina, Salvador Bahia \\ ${ }^{2}$ Programa de Pós-Graduação em Mecatrônica (PPGM) \\ Av. Adhemar de barros, s/n, Ondina, Salvador, Bahia, CEP: 40170110 \\ dclaro@ufba.br
}

\begin{abstract}
Heterogeneous devices in IoT need to communicate and web services can provide such interoperability. However, it is necessary to embed those web services on such devices. Our work describes the development of the course MAT700 at the Federal University of Bahia aimed to provide knowledge to interoperate heterogeneous devices by web services. Finally, we evaluated various aspects related to the course, including learning, content, didactics, in order to validate our proposed interdisciplinary methodology. The results showed that the interdisciplinary contributed to the individual learning and growth learners.
\end{abstract}

Keywords: heterogeneous devices, interdisciplinary, web services

\section{Introdução}

Atualmente muito se tem discutido a respeito da Internet das Coisas (Internet of Things - IoT), cujo propósito está na conexão de "coisas" do mundo físico ao mundo da Internet de forma que estes passem a ser reconhecidos e tratados como qualquer recurso. Estes recursos têm utilizado protocolos e padrões já existentes, como por exemplo HTTP (Hypertext Transfer Protocol) e URIs (Uniform Resource Identifier) (Guinard, 2010).

A possibilidade de inserção de diferentes dispositivos físicos ("coisas") na Web representa um cenário propício para utilização dos dados e serviços compartilhados por eles na concepção de novas aplicações. Um fator relevante, que de certa forma pode se tornar impeditivo nesse contexto está atrelado ao formato de como eles se conectam a Internet, já que, em sua maioria, esta conectividade é subsidiada por softwares dedicados e interfaces proprietárias (Guinard, 2010). Em se tratando dos mesmos fornecedores, a comunicação entre dispositivos muitas vezes ocorre de maneira fácil e rápida. Com o avanço e a proliferação da Internet das Coisas, novos dispositivos estão sendo incorporados na rede, independente de fabricante, o que vem dificultando a comunicação e consequentemente a interoperabilidade entre estes dispositivos. Neste sentido, interoperar dois dispositivos distintos com o intuito de que haja uma mínima comunicação entre eles, tem se tornado uma tarefa árdua para a maioria dos desenvolvedores. 
V Congresso Brasileiro de Informática na Educação (CBIE 2016)

Anais do XXII Workshop de Informática na Escola (WIE 2016)

Os serviços web têm por principal característica garantir a interoperabilidade através de interfaces e troca de mensagens padronizadas (Claro, Albers, \& Hao, 2007). Assim, embarcar serviços web nos dispositivos permite que estes sejam tratados como serviços web, facilitando a comunicação entre dispositivos heterogêneos e garantindo assim a interoperabilidade.

Diante deste contexto, a disciplina de Tópicos Especiais em Mecatrônica do Mestrado em Mecatrônica da Universidade Federal da Bahia foi proposta com o intuito de desenvolver um estudo para embarcar serviços web em dispositivos da Internet das Coisas. Por característica, este curso tem duas principais linhas de pesquisa: a Ciência da Computação e a Mecatrônica, tendo consequentemente discentes matriculados de ambas as áreas. Estes discentes podem ter diversas formações, tais como Ciência da Computação, Sistemas de Informação, Engenharia Mecânica, Engenharia da Computação, Engenharia Elétrica, e áreas afins. A disciplina foi dividida em etapas culminando com o desenvolvimento de um serviço web embarcado a fim de interoperar dispositivos distintos. O principal objetivo deste trabalho é apresentar o processo de desenvolvimento da disciplina, analisar os resultados obtidos e avaliar a aprendizagem em se tratando de uma turma interdisciplinar com conhecimentos fundamentais diferentes, mas relevantes para $\mathrm{o}$ desenvolvimento do trabalho. A principal hipótese deste trabalho é que a interdisciplinaridade contribui e motiva o aprendizado em turmas heterogêneas. Especificamente descreveu-se a metodologia proposta, os experimentos realizados pelos discentes e a avaliação sobre a didática e a aprendizagem durante o período dos experimentos e em relação à hipótese mencionada.

Este artigo está assim organizado. A seção 2 apresenta a metodologia utilizada no desenvolvimento da disciplina, a seção 3 apresenta a descrição de cada experimento proposto pelas equipes de discentes, a seção 4 apresenta os resultados em relação à didática, metodologia e aprendizagem e a seção 5 apresenta algumas conclusões em relação aos resultados obtidos.

\section{Metodologia}

Este trabalho foi desenvolvido no contexto da disciplina MAT700 de Pós-Graduação da UFBA com o intuito de interoperar dispositivos heterogêneos. A turma foi caracterizada por alunos heterogêneos, cuja as formações correspondiam a Ciência da Computação (CC), Sistemas de Informação (SI), Engenharia da Computação (EC), Engenharia Mecânica (EM) e Engenharia Elétrica (EE). Os discentes foram divididos em duplas e estas duplas eram interdisciplinares, ou seja, um aluno da linha de pesquisa em Ciência da Computação (CC e SI) e outro aluno na linha de Mecatrônica (EC, EM, EE). O principal objetivo desta organização foi a troca de conhecimento especifico em cada área, necessária para o desenvolvimento do trabalho final. Ao total foram 5 duplas, totalizando 10 alunos.

A disciplina foi dividida em 5 macro etapas: i) estudo dos conceitos básicos, tais como Internet das Coisas, Serviços Web e Dispositivos; ii) conhecimento básico na placa Raspberry Pi e iii) conhecimento básico em um serviço web; iv) desenvolvimento de um serviço web embarcado e v) interoperabilidade entre dispositivos heterogêneos.

Em relação ao estudo dos conceitos básicos, foi proposto que cada dupla desenvolvesse slides sobre cada um dos tópicos e estes seriam apresentados pela dupla. No que concerne o conhecimento básico de uma placa, foi proposto o desenvolvimento de um circuito 
V Congresso Brasileiro de Informática na Educação (CBIE 2016)

Anais do XXII Workshop de Informática na Escola (WIE 2016)

elétrico para acender um led através da placa Raspberry Pi e da protoboard, no qual os membros da Mecatrônica contribuíram mais enfaticamente devido ao notório conhecimento neste tema. Por outro lado, em se tratando dos serviços web, foi proposto o desenvolvimento de um serviço web simples somente para garantir a troca de mensagens. Neste caso, os discentes da Ciência da Computação contribuíram com mais profundidade por se tratar de um tema mais intrínseco a sua área. E por fim, foi embarcado o serviço web desenvolvido na placa Raspberry Pi com o intuito de garantir que era factível embarcar em um dispositivo com características reduzidas, tais como: pouco espaço de armazenamento, baixo processamento e pouca memória.

Em relação ao trabalho final proposto, o principal desafio foi embarcar serviços web em dispositivos tais como placas Raspberry Pi modelo B para domínios distintos que se comunicassem com dispositivos distintos. Para tal, os discentes tinham como requisito utilizar uma destas linguagens: Java, Python ou Node JS e tratar de um domínio distinto do apresentado em sala de aula. Os discentes foram estimulados a desenvolver uma aplicação que permitisse analisar a interoperabilidade entre dois dispositivos distintos, garantindo assim a troca de mensagens.

Cada dupla ficou responsável pelo desenvolvimento de um serviço web embarcado em uma placa Raspberry independente de domínio. A independência de domínio era responsável pela motivação da aplicação a ser desenvolvida, resguardando o principal propósito do trabalho: aprendizagem sobre o desenvolvimento de um serviço web embarcado em uma placa garantindo a interoperabilidade entre dispositivos distintos. Os experimentos e resultados são apresentados nas próximas seções deste trabalho.

\section{Experimentos com as equipes interdisciplinares}

Cada dupla desenvolveu o seu experimento utilizando uma Raspberry Pi de $900 \mathrm{MHz}$ quad-core ARM Cortex-A7, 1 GB RAM, MicroSDHC slot, contendo um SD Card de 16 GB, Classe 4(Pi, 2016) (Upton \& Halfacree, 2014).

\subsection{Gerenciamento de espaço de eventos}

Este primeiro experimento teve por objetivo gerenciar os espaços de eventos, baseado em casos que ultrapassam limites permitidos, tais como: horário limite, volume do som, gestão de entrada, lâmpadas e demais dispositivos mapeados no ambiente. Dois serviços web distintos foram desenvolvidos: 1) desligar e ligar tensão de uma rede elétrica simulada por uma lâmpada e 2) acionar um conjunto de refletores com LED RGB no espaço de evento.

\subsubsection{Materiais}

A Raspberry Pi 2 foi utilizada como servidor central e na simulação do conjunto de refletores foi utilizada um Led RGB de $5 \mathrm{~mm}$ de 4 terminais com anodo comum. Um circuito com módulo relé ${ }^{1}$ para o acionamento de cargas de alta tensão para simulação de lâmpadas e ventiladores ligados em uma rede $\mathrm{AC}$ de $110 \mathrm{~V}$ ou $220 \mathrm{~V}$ foi implementado. O Raspbian, sistema operacional baseado no Debian, foi utilizado juntamente com o

\footnotetext{
${ }^{1}$ O módulo relé (relay) é composto por dispositivos comutadores eletromecânicos, os quais contêm um eletroímã e uma armadura móvel que tem por finalidade abrir ou fechar um jogo de contatos (1, nao).
} 
V Congresso Brasileiro de Informática na Educação (CBIE 2016)

Anais do XXII Workshop de Informática na Escola (WIE 2016)

servidor apache tomcat 7, contendo a biblioteca jersey para serviço web RESTful (Jersey, 2016) e a biblioteca pi4j para manipulação da Raspberry $P i$.

\subsubsection{Métodos}

Dois serviços RESTful distintos foram desenvolvidos, um para o controle da lâmpada e outro para o led RGB. No serviço da lâmpada foram definidas três chamadas utilizando as requisições HTTP: 1) no método GET que retorna o status da lâmpada, 2) no método POST responsável por ligar a lâmpada e 3) no método DELETE, responsável por desligar a lâmpada.

Na planta de simulação para que aconteça o acionamento do Led, é necessário que o pino de GPIO da Raspberry Pi esteja em estado baixo (low/0) pois o mesmo sempre está alimentando em $5 \mathrm{~V}$.

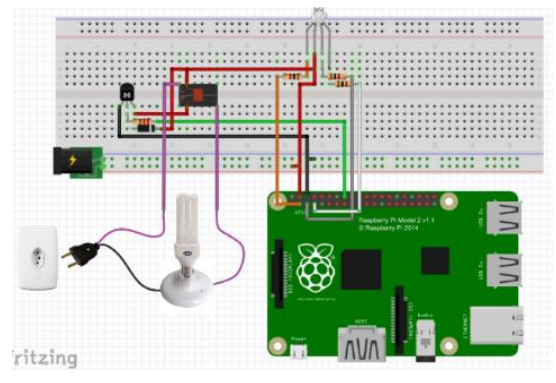

Figura 1: Esquemático de ligação de componentes para o experimento de gerenciamento de espaço de eventos (Knörig \& Howell, 2010).

Na realização de testes foi utilizada a GPIO1 da Raspberry para comandar um módulo relé acoplado a uma lâmpada incandescente ligada a rede elétrica de $110 \mathrm{~V}$, a GPIO2 para o acionamento da cor vermelha, GPIO3 para a cor verde e a GPIO4 para cor azul do LED, como o diagrama demonstrado na Figura 1.

\subsection{Semáforo Web}

Uma segunda proposta de trabalho de outra equipe foi o desenvolvimento de um sistema de semáforos inteligentes para o controle de tráfego através de serviços Web.

\subsubsection{Materiais}

O desenvolvimento do semáforo utilizou as pinagens da Raspberry Pi (GPIO; GROUND; $5 \mathrm{~V} ; 3.3 \mathrm{~V})$ capaz de integrar o mundo da computação com o mundo real. Analisou-se conceitos como corrente, tensão, controle digital, resistências e diodo emissor de luz (LED). A linguagem Python foi utilizada em conjunto com o framework Web.py ${ }^{2}$. O circuito foi desenvolvido com três LED's (verde, amarelo, vermelho) para a simulação de um semáforo como se observa na Figura 2.

\subsubsection{Métodos}

O código desenvolvido contém inicialmente os nomes dos pinos a serem utilizados, a configuração inicial das portas GPIO e a sequência de acendimento dos LED's. Na figura 3 são apresentados os estados do semáforo após seu acionamento. O semáforo controlado 
V Congresso Brasileiro de Informática na Educação (CBIE 2016)

Anais do XXII Workshop de Informática na Escola (WIE 2016)

pela web abre possibilidades para variar as luzes em momentos livres ou durante uma emergência.

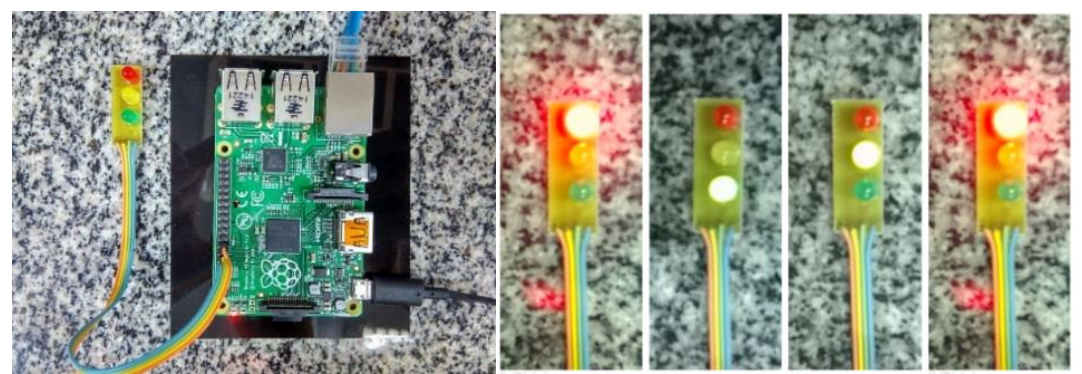

Figura 2: Circuito incluindo Raspberry PiB+ e o semáforo para testes ligado nas saídas GPIO 17, 27 e 22, verde, amarelo e vermelho respetivamente

\subsection{Keep Iced}

O terceiro experimento proposto visa monitorar a temperatura interna de qualquer tipo de cooler armazenador de bebidas e avisar ao usuário quando deverá ser inserido mais gelo no recipiente através de um aplicativo para smartphones.

\subsubsection{Materiais}

O protótipo é baseado no sensor de temperatura e umidade DHT11; um resistor de $5 \mathrm{~K} \Omega$; uma protoboard; um cooler; um smartphone com tecnologia Android; e, um computador para o desenvolvimento do código.

\subsubsection{Métodos}

Na protoboard foi montado o circuito especificado no datasheet disponibilizado pelo fabricante do sensor DHT11 e com a ajuda das bibliotecas Restify e Node-DHT-Sensor, foi desenvolvido um código em JavaScript para a criação do servidor REST, o qual tornou possível a leitura dos dados de temperatura e umidade do sensor, e o acesso a esses valores através da internet ou rede local.

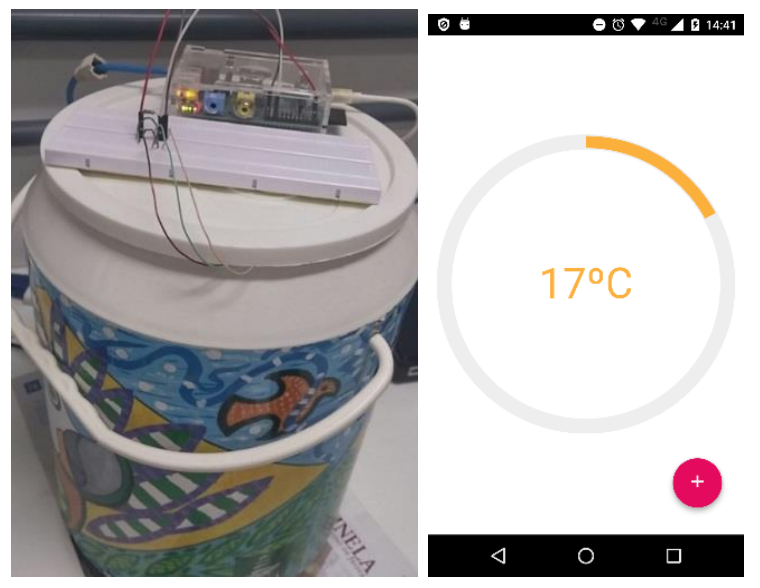

Figura 3. Protótipo finalizado e tela inicial do aplicativo.

Um aplicativo para smartphones foi desenvolvido com tecnologia híbrida com o intuito de se comunicar com o dispositivo. Este aplicativo captura a informação de temperatura do sensor através do serviço disponibilizado pelo servidor, na rota especificada acima, utilizando o método GET do protocolo HTTP. A Figura 3, mostra o protótipo finalizado e a tela de leitura do sensor de temperatura, disponível no aplicativo. 
V Congresso Brasileiro de Informática na Educação (CBIE 2016)

Anais do XXII Workshop de Informática na Escola (WIE 2016)

\subsection{Braço Robótico}

Este quarto experimento foi responsável por manusear um braço robótico que manipula objetos e os coloca em cima de um chassi de veículo. Este chassi possui um sensor de ultrassom para detectar os objetos colocados de modo incorreto e que, portanto, ultrapassaram os limites do chassi.

\subsubsection{Materiais}

Os principais componentes foram: placa Raspberry P $i$, um Arduino uno, um sensor de ultrassom, 4 servo-motores do braço robótico, um roteador $4 \mathrm{G}$ sem fio e um adaptador sem fio para conexão dos dispositivos na Internet e 2 serviços web implementados no Node.JS.

\subsubsection{Métodos}

Dois serviços web foram implementados. O primeiro foi embarcado no braço robótico para controlar os movimentos do braço e receber as requisições de um usuário sobre a existência de novas peças. Enquanto que o segundo, denominado detector de objetos nos limites do chassi, foi implementado para recuperar os dados do sensor de ultrassom e receber também requisições do usuário, conforme ilustrado na Figura 4.

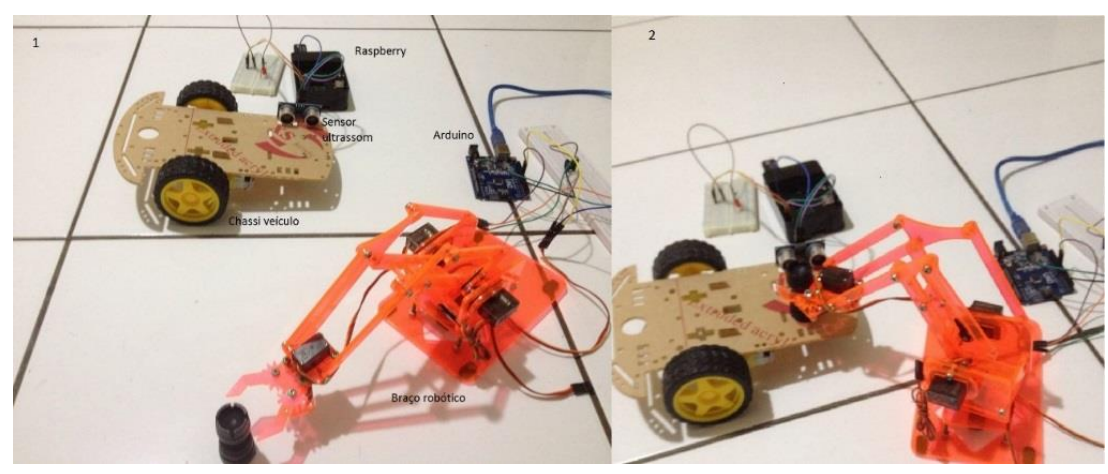

Figura 4. Cenário do braço robótico

O usuário solicita que o serviço web do braço robótico manipule um objeto e o coloque em cima do chassi. Após a colocação do objeto, o serviço web do ultrassom é requisitado para saber se o objeto foi colocado corretamente. A Figura 4 demonstra as duas etapas executadas: a) o braço robótico captura o objeto através da sua garra e 2) coloca o objeto em cima do chassi do veículo. O funcionamento correto do braço robótico acarreta na colocação adequada dos objetos no chassi sem interferir nos seus limites.

\subsection{Monitoramento de um ambiente}

Neste quinto experimento foi proposto um monitoramento de um ambiente, incluindo as medidas de temperatura, umidade e detector de presença.

\subsubsection{Materiais}

Os materiais utilizados foram: um sensor de temperatura e umidade (DHT11) e um sensor que mensura a distância (HC-SR04).

\subsubsection{Métodos}


V Congresso Brasileiro de Informática na Educação (CBIE 2016)

Anais do XXII Workshop de Informática na Escola (WIE 2016)

Os circuitos foram habilitados para a coleta de dados dos sensores. Na Figura 5 (a), observa-se o circuito final montado, em protoboard, para o sensor de distância (HCSR04). Já na Figura 5 (b), observa-se o circuito final montado, em protoboard, para o sensor de temperatura e umidade (DHT11).

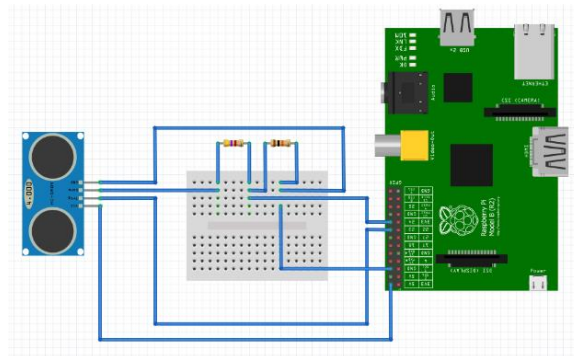

(a) Circuito Sensor $\mathrm{HC}-\mathrm{SRO4}$

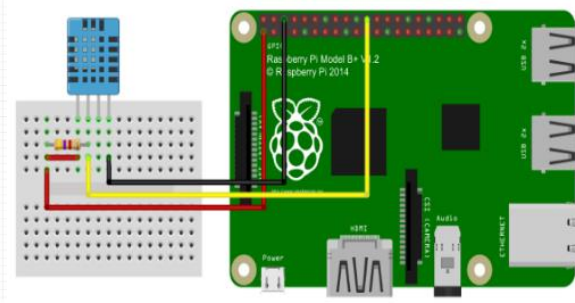

(b) Circuito Sensor DHT11

Figura 5 - Circuitos em Protoboard para monitoramento do ambiente (Knörig \& Howell, 2010)

Em seguida, utilizaram-se dois scripts em Python, um para o sensor DHT11 e outro para o sensor HC-SR04. Após verificação dos sensores, os serviços web RESTful coletaram os dados de leitura do sensor de temperatura e umidade. Enquanto que para o sensor de distância, utilizou-se o protocolo SOAP.

\section{Avaliação da Aprendizagem}

A avaliação da aprendizagem ocorreu em dois momentos distintos. Primeiramente, ao final das apresentações, foi feita uma auto-avaliação no quadro sobre o processo de aprendizagem, envolvendo os aspectos positivos e negativos desde o primeiro dia de aula até a conclusão destas apresentações. Em um segundo momento, foi elaborado um questionário avaliativo no qual os alunos responderam e os resultados são apresentados nas seções seguintes.

\subsection{Auto-avaliação no Quadro}

Nesta auto-avaliação (Figura 6), primeiramente buscou-se determinar os conteúdos aprendidos com o desenvolvimento da disciplina e quais destes tinham sido mais absorvidos pelos alunos. Os conteúdos elencados por todos foram divididos em 5 categorias: a) parte eletrônica (placa Raspberry Pi, GPIO, Sensores e Arduino), b) serviços web, os quais os alunos destacaram os serviços implementados utilizando o protocolo SOAP e RESTful, c) padronização das requisições, na qual foi destacada a linguagem de definição de interface dos serviços web, WSDL, tanto para SOAP quanto para RESTful, d) programação, que envolveu as três principais linguagens de desenvolvimento de serviços web, Python, Javascript e Java. E por fim, a e) Web das Coisas, como o principal ambiente para manuseio dos dispositivos desenvolvidos.

Em seguida, objetivou-se analisar a adequação da metodologia proposta no desenvolvimento dos projetos, onde se procurou descrever os pontos positivos e os pontos negativos observados. Dentre os pontos positivos, destacou-se o aprendizado; a interdisciplinaridade, observando que eram necessários conhecimentos de ambas as áreas computação e mecatrônica; a inovação, visto que os alunos tinham a flexibilidade de propor projetos dentro do seu domínio de interesse.

Em relação aos pontos negativos, os alunos pontuaram o tempo para o desenvolvimento dos projetos e a falta de uma infraestrutura adequada, por exemplo, uma bancada 
V Congresso Brasileiro de Informática na Educação (CBIE 2016)

Anais do XXII Workshop de Informática na Escola (WIE 2016)

eletrônica, maior quantidade de placas Raspberry Pidisponíveis por aluno, maior quantidade de sensores, etc.

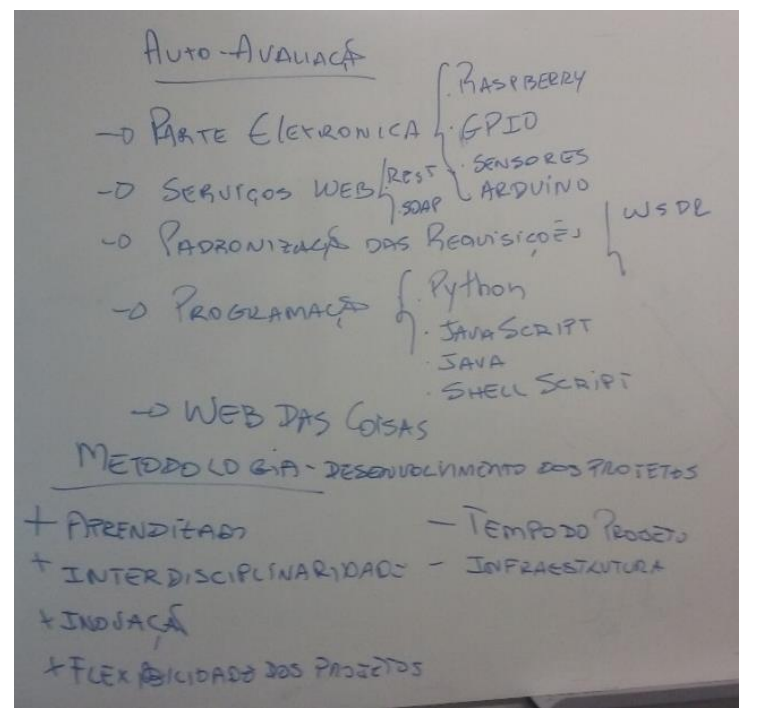

Figura 6 - Auto-avaliação no dia das apresentações

Em seguida, foi elaborado um questionário mais especifico sobre aprendizagem e didática que foi aplicado aos alunos.

\subsection{Avaliação através de questionário}

A avaliação através deste questionário ocorreu com o objetivo de avaliar outros aspectos além do conteúdo ministrado. Primeiramente, foi avaliado o 1) nível de esforço para acompanhar a disciplina, com o intuito de verificar se a interdisciplinaridade proposta tinha tido algum impacto negativo. Em seguida, foi analisado a 2) contribuição para o aprendizado do aluno, comparando o nível de conhecimento no assunto no início do curso e o seu nível de conhecimento no final do curso. Analisou-se também a 3) competência e responsabilidade do professor que estava ministrando a disciplina, envolvendo aspectos desde o conhecimento no assunto até sua postura em sala de aula. No item 4) observouse o conteúdo do curso, em relação aos objetivos, organização, carga de trabalho. No item 5) foi analisado o interesse do aluno na disciplina, se estavam por obrigação de carga horaria ou se estavam porque tinham interesse no tema. E por fim, dois campos em texto livre foram analisados sobre quais os aspectos do curso foram mais uteis e importantes para o aprendizado do aluno e o que o aluno gostaria que fosse melhorado no curso.

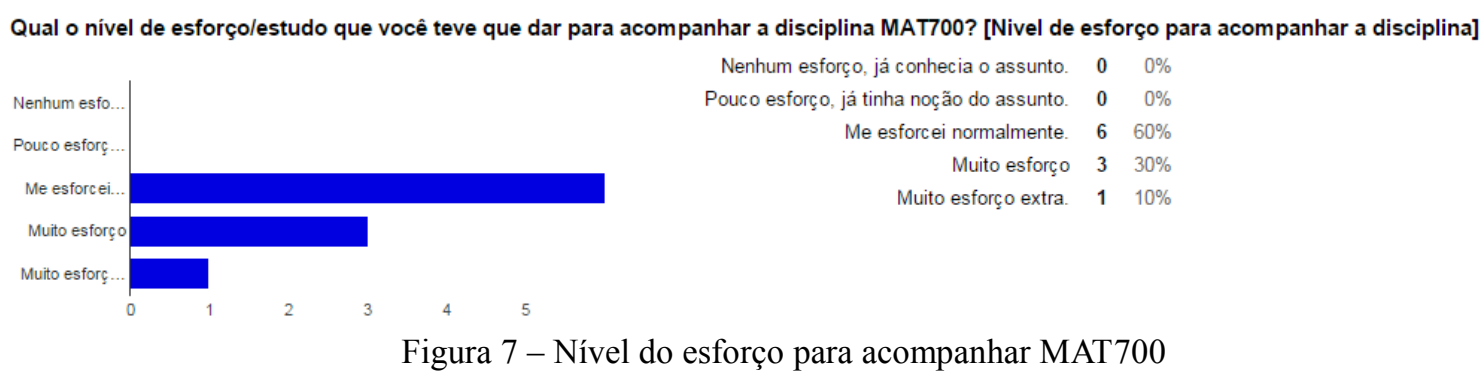

Como resultados em relação ao item 1 , referente ao nível de esforço que cada aluno (Figura 7), observa-se que a maioria dos alunos informaram que se esforçou normalmente, evidenciando que a interdisciplinaridade correspondeu a um aspecto positivo da proposta. 
V Congresso Brasileiro de Informática na Educação (CBIE 2016)

Anais do XXII Workshop de Informática na Escola (WIE 2016)

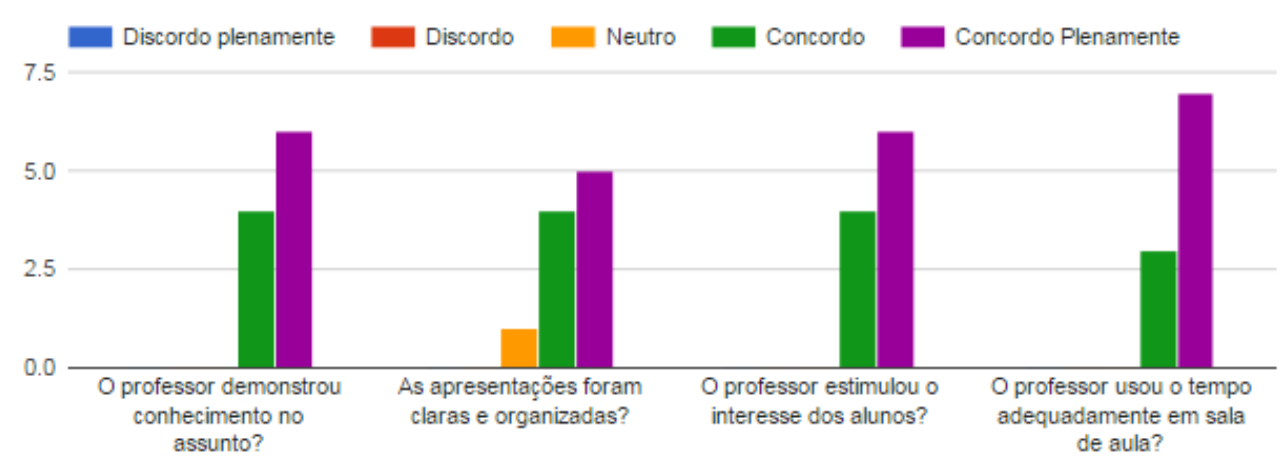

Figura 9 - Competência e Responsabilidade do docente

Em relação a contribuição para o aprendizado (item2), foi analisado o conhecimento antes do curso e depois do curso ministrado. Neste sentido, a Figura 8 apresenta que no início do curso, se tinha pouco conhecimento no assunto (gráfico laranja) enquanto que no final do curso, observou-se que o conhecimento tinha sido atingido (gráfico vermelho). O gráfico verde informou que curso contribuiu muito para as habilidades e conhecimentos dos alunos.

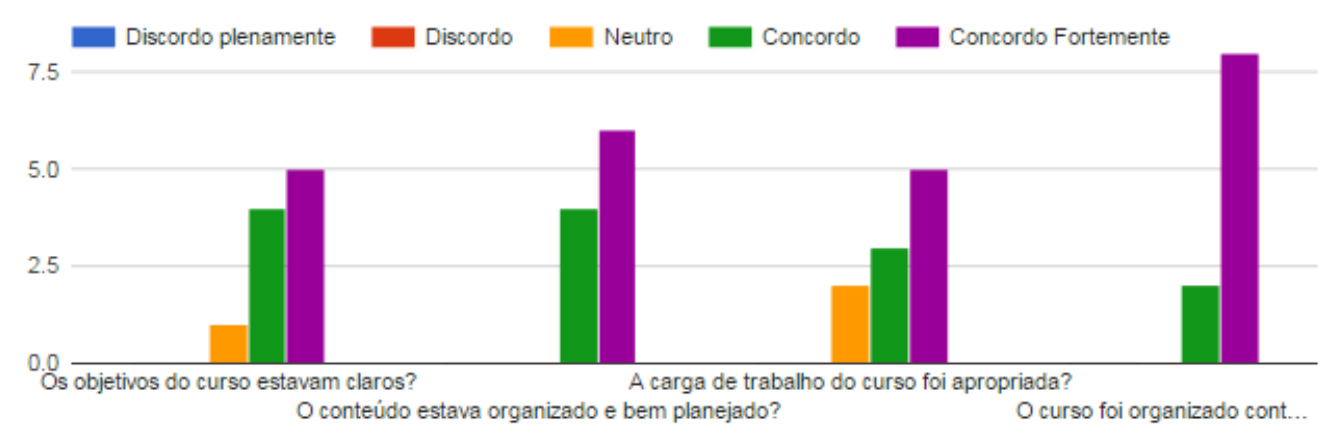

Figura 10 - Conteúdo do curso

Em relação ao item 3, a competência e responsabilidade do docente também foi avaliada. A figura 9 apresenta que o professor teve comprometimento com a disciplina, tinha domínio do conteúdo e ainda se observou que o mesmo esteve disponível e pronto a ajudar em $100 \%$ dos casos.

O item 4 correspondeu ao conteúdo da disciplina que conforme a Figura 10 observou-se que o conteúdo estava bem organizado, claro e que contemplou a participação de todos os estudantes. O item 5 analisou o interesse dos alunos na disciplina, onde a maioria demonstrou interesse no tema a ser abordado.

E por fim, em se tratando das questões abertas, a primeira em relação ao conteúdo mais útil, as respostas foram divididas entre manipulação da placa Raspberry $\mathrm{e}$ desenvolvimento de serviços web e a IoT. Na segunda, como eles gostariam que o curso melhorasse, a maioria sugeriu mais aulas práticas para a manipulação dos dispositivos.

\section{Conclusões}

A interoperabilidade de dispositivos através do uso de serviços web permite que diversos dispositivos da IoT se comuniquem trocando mensagens padronizadas e garantindo a integração de dispositivos distintos. Com os trabalhos apresentados nesta disciplina, além do conhecimento adquirido sobre serviços web embarcados em dispositivos, conforme 
V Congresso Brasileiro de Informática na Educação (CBIE 2016)

Anais do XXII Workshop de Informática na Escola (WIE 2016)

demonstraram as avaliações, já houve a submissão de projetos oriundos desta disciplina para editais de inovação.
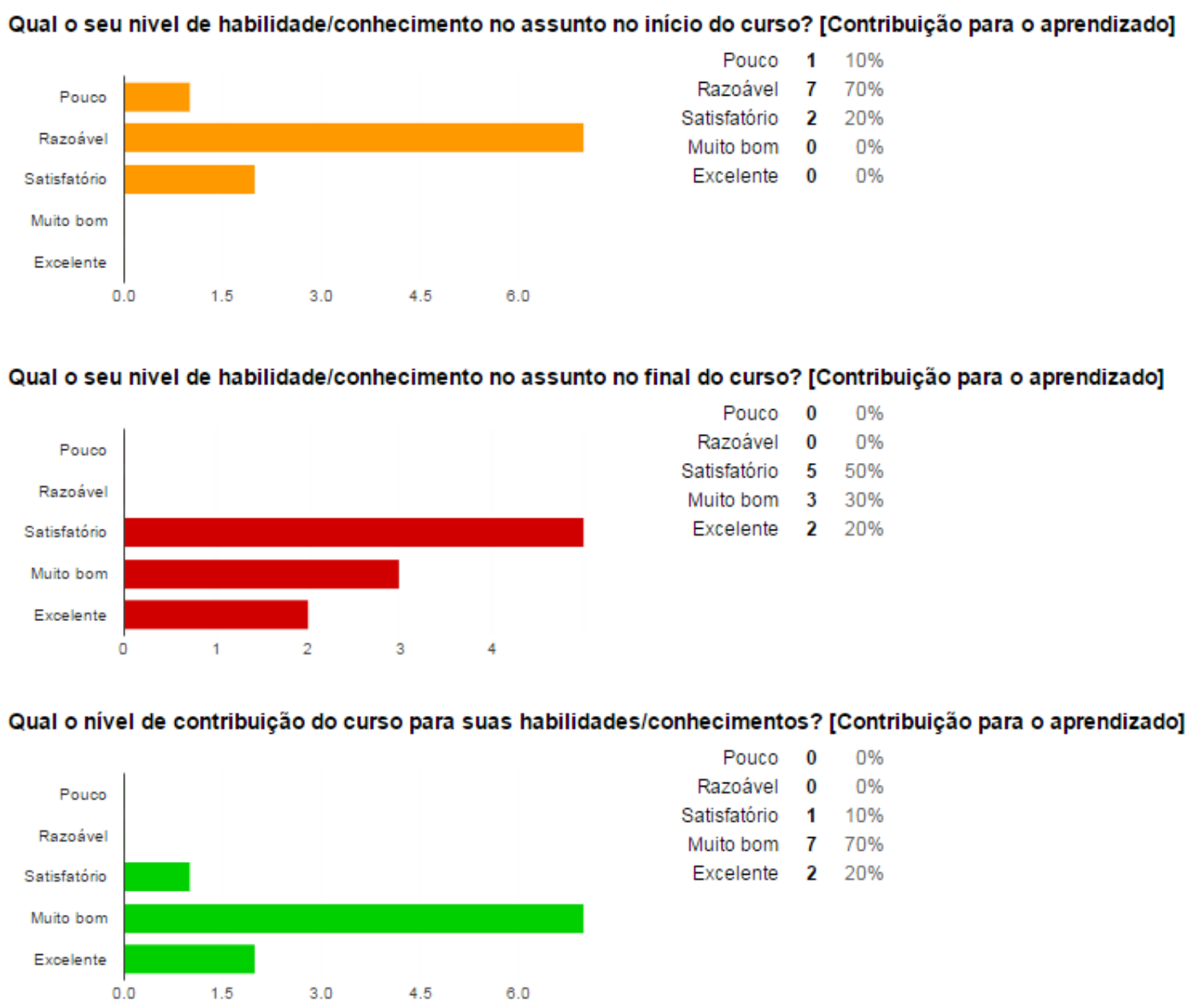

Figura 8 - Contribuição para o aprendizado com MAT700

Assim, observou-se que tanto o aprendizado quanto o alicerce foram contemplados, dando origem a novos projetos e novas possibilidades de troca de conhecimento interdisciplinar. $\mathrm{O}$ interesse pelo tema e a flexibilidade dos trabalhos, foram fatores essenciais para a criatividade e motivação dos alunos no desenvolvimento da disciplina.

\section{Referências}

Claro, D. B., Albers, P., \& Hao, J.-K. (2007). A Framework for Automatic Composition of RFQ Web Services. Proceedings of the First Workshop on Web Service Composition and Adaptation (WSCA) held in conjunction with IEEE International Conference of Web Services (ICWS'07) (pp. 221-228). Salt Lake City: IEEE.

Guinard, D. (2010). Towards Opportunistic Applications in a Web of Things. IEEE International Conference on Pervasive Computing and Communications Workshops, (pp. 863-864).

Jersey, A. (2016). RESTful Web Services in Java. Fonte: https://jersey.java.net. Acessado em 31/05/16

Knörig, A., \& Howell, B. (2010). Advanced prototyping with Fritzing. Proceedings of Tangible, Embodied and Embedded Interaction 2010. Boston: USA.

Nunes, L., L.H.V., N., Libardi, R., Estrella, J., Santana, M., \& Santana, R. (2014). Análise de Desempenho em Dispositivos Limitados e Emulados. Estudo de caso: Raspberry Pie Web Services RESTful. WPerformance - XIII Workshop em desempenho de Sistemas Computacionais e de Comunicação. Brasilia: SBC.

Pi, R. (2016). What is a Raspberry Pi? Fonte: www.Raspberrypi.org. Acessado em 31/05/2016

Upton, E., \& Halfacree, G. (2014). Raspberry Pi user guide. USA: Jonh Wiley \& Sons. 